Taxonomía y sistemática

\title{
Nuevos registros de hongos entomopatógenos del género Cordyceps $s . l$. (Ascomycota: Hypocreales) del Estado de México
}

\author{
New records of entomopathogenous fungi of the genus Cordyceps s. 1. (Ascomycota: Hypocreales) \\ from the State of México
Juan Carlos Pérez-Villamares ${ }^{\text {a }}$, Cristina Burrola-Aguilar ${ }^{\text {a,* }}$, Xóchitl Aguilar-Miguel ${ }^{\text {a }}$, Tatiana Sanjuan ${ }^{\mathrm{b}}$ y Esteban Jiménez-Sánchez ${ }^{\mathrm{c}}$
${ }^{a}$ Centro de Investigación en Recursos Bióticos, Facultad de Ciencias, Universidad Autónoma del Estado de México, carretera Toluca-Atlacomulco, Km 14.5, 50200, Toluca, Estado de México, México
${ }^{\text {b } L a b o r a t o r i o ~ d e ~ M i c o l o g i ́ a ~ y ~ F i t o p a t o l o g i ́ a, ~ U n i v e r s i d a d ~ d e ~ l o s ~ A n d e s, ~ C a r r e r a ~} 1$ Núm. 18a -12, Bogotá 111711, Colombia
${ }^{\text {c }}$ Laboratorio de Zoología, Facultad de Estudios Superiores Iztacala, Universidad Nacional Autónoma de México, Av. de los Barrios No.1, Los Reyes Iztacala, 54090, Tlalnepantla, Estado de México, México \\ Recibido el 17 de noviembre de 2015; aceptado el 23 de mayo de 2017 \\ Disponible en Internet el 14 de noviembre de 2017
}

\section{Resumen}

Cordyceps $s$. l. es un género de hongos entomopatógenos muy poco estudiado en México, y debido a las escasas exploraciones se desconoce la diversidad de especies presentes en la región. Con el objetivo de contribuir al conocimiento de este grupo taxonómico, se realizaron recolectas en el sureste del Estado de México para determinar las especies de Cordyceps s. l. presentes en la región. Se examinaron en total 236 especímenes que se agrupan en 7 especies identificadas: Ophiocordyceps entomorrhiza, O. gracilioides, O. gracilis y la fase conidial de Ophiocordyceps stylophora, que son nuevos registros para el Estado de México, y Cordyceps takaomontana y C. scarabaeicola, las cuales se citan por primera vez para México. Con este estudio que incrementan a 17 las especies registradas para México, que es considerado uno de los 5 países megadiversos del planeta, se demuestra la necesidad de realizar nuevas exploraciones para la identificación de hongos entomopatógenos.

(C) 2017 Universidad Nacional Autónoma de México, Instituto de Biología. Este es un artículo Open Access bajo la licencia CC BY-NC-ND (http://creativecommons.org/licenses/by-nc-nd/4.0/).

Palabras clave: Cordycipitaceae; Ophiocordycipitaceae; Cordyceps scarabaeicola; Ophiocordyceps gracilis; Lepidoptera; Coleoptera

\begin{abstract} need for further exploration on this specific taxonomic group of fungi. (http://creativecommons.org/licenses/by-nc-nd/4.0/).

\footnotetext{
* Autor para correspondencia.

Correo electrónico: cba@uaemex.mx (C. Burrola-Aguilar).

La revisión por pares es responsabilidad de la Universidad Nacional Autónoma de México.
}

Cordyceps s. $l$. is an entomopathogenic genus that has been poorly studied in Mexico and because of the limited explorations, the species diversity of this group in the region is unknown. Therefore, with the objective to contribute to the knowledge of this taxonomic group, samplings were carried out in the Southeastern region of the State of México to determine the diversity of the genus. In total 236 specimens were examined, which represent 7 identified species: Ophiocordyceps entomorrhiza, O. gracilioides, O. gracilis and the conidial stage of Ophiocordyceps stylophora, which are first records for the State of México, and Cordyceps takaomontana and C. scarabaeicola, first records for Mexico. This new research increases the records of entomopathogenous fungi to 17 known species for Mexico, one of the 5 megadiverse countries in the world, showing the

(C) 2017 Universidad Nacional Autónoma de México, Instituto de Biología. This is an open access article under the CC BY-NC-ND license

Keywords: Cordycipitaceae; Ophiocordycipitaceae; Cordyceps scarabaeicola; Ophiocordyceps gracilis; Lepidoptera; Coleoptera 


\section{Introducción}

Cordyceps Fr. sensu lato (s. l.) (Ascomycota: Hypocreales) es un género de hongos parásitos de invertebrados muy diverso (Sung et al., 2007). Está constituido por más de 500 especies (Index Fungorum, 2015), las cuales se distribuyen principalmente en regiones tropicales de Asia y América (Kobayasi, 1941; Mains, 1958; Sung et al., 2007). Las especies de Cordyceps $s$. $l$. tienen una amplia variedad de hospederos; la mayoría son parásitas de insectos de varios órdenes y de arácnidos, y también un reducido número de especies son parásitas de hongos del género Elaphomyces Nees (Nikoh y Fukatsu, 2000). Las especies de Cordyceps s. $l$. se han utilizado por muchos años en la medicina tradicional de países asiáticos como China, Corea y Japón (Wen, Li, Kang y He, 2012) y se ha comprobado que poseen componentes bioactivos benéficos para la salud humana como la cordicepina, un metabolito secundario con propiedades anticancerígenas, antioxidantes y antiinflamatorias (Tuli, Sandhu y Sharma, 2014).

El estudio taxonómico de Cordyceps s. $l$. ha sido muy complejo desde que se comenzó a estudiar en el siglo XIX. Habitualmente este género se incluía solo dentro de la familia Clavicipitaceae (Kobayasi, 1941, 1982; Mains, 1958). Recientemente se realizó un estudio filogenético con datos moleculares, a partir del cual se planteó una nueva clasificación en la que separa al género Cordyceps en 3 familias y 4 géneros: Cordyceps sensu stricto (s. $s$ ) (Cordycipitaceae), incluyendo especies anamórficas de los géneros Beauveria Vuill., Isaria Pers. y Lecanicillium W. Gams et Zare (Sung et al., 2007); Metacordyceps G.H. Sung, J.M. Sung, Hywel-Jones et Spatafora, y las especies anamórficas del género Metarhizium Sorokin (Sung et al., 2007). La familia Ophiocordycipitaceae agrupa a los géneros Elaphocordyceps G.H. Sung et Spatafora y Ophiocordyceps Petch emend G.H. Sung, J.M. Sung, Hywel-Jones et Spatafora (Sung et al., 2007). Elaphocordyceps está asociado a especies anamórficas del género Tolypocladium W. Gams, mientras que Ophiocordyceps incluye anamorfos de los géneros Hirsutella Pat., Hymenostilbe Petch, Paraisaria Samson et B. L. Brady y Syngliocladium Petch. (Sung et al., 2007). De acuerdo con Hibbett y Taylor (2013), se ha propuesto unificar con un solo nombre a las diferentes formas teleomórficas y anamórficas de la misma especie de hongo. Al respecto, los géneros teleomorfos $\mathrm{y}$ anamorfos que se han reconciliado bajo un mismo nombre son: Metacordyceps, el cual se ha suprimido para preservar el nombre genérico Metarhizium (Kepler, Humber, Bischoff y Rehner, 2014) y Elaphocordyceps, el cual se ha suprimido conservando el nombre genérico más antiguo Tolypocladium (Quandt et al., 2014). Para el caso de Cordyceps s. s. y Ophiocordyceps no se han hecho cambios con respecto a sus especies anamórficas.

El género Cordyceps s. $l$. ha sido poco estudiado en México: hasta la fecha se han descrito 15 especies (Guzmán, Morón, Ramírez-Guillén y Wolf, 2001). Para el Estado de México se cuenta con un solo registro de la especie entomopatógena Cordyceps militaris (Frutis, Chio y Estrada-Torres, 1985) y 2 especies parásitas de Elaphomyces: Tolypocladium capitatum (Holmsjold) Quandt, Kepler et Spatafora (como Clavaria capitata Holmskjold) y Tolypocladium ophioglossoides Quandt, Kepler et Spataphora (como Cordyceps ophioglossoides [Ehrh], Link) (Heim y Wasson, 1958).

El escaso conocimiento sobre la diversidad y la taxonomía de las especies de hongos entomopatógenos del género Cordyceps $s$. $l$. en México es el reflejo de la falta de exploraciones en campo para este grupo de hongos en particular. Por lo tanto, el objetivo del presente estudio es contribuir al conocimiento de estos hongos a partir de nuevas exploraciones en diferentes localidades del Estado de México con la finalidad de aportar nuevos datos de diversidad y taxonomía de las especies de Cordyceps s. l. presentes en México.

\section{Materiales y métodos}

Se realizaron 22 exploraciones semanales durante la época de lluvias, de julio a noviembre de 2013 y 2014, en 3 tipos de vegetación de 5 localidades del sureste del Estado de México: Malinalco, Ocuilan, Tenancingo, Tenango del Valle y Villa Guerrero. Para el análisis macroscópico de los especímenes se consideraron los datos en fresco de tamaño del estroma, el color y la posición de los peritecios dentro del tejido estromático. La determinación del color se obtuvo del sitio en línea HTML Color Codes (2016) (http://html-color-codes.info/). Para el análisis microscópico de los especímenes se obtuvo una muestra del tejido fértil, la cual se tiñó en rojo Congo al 10\% para observar peritecios, ascas, ascosporas o conidióforos, fiálides y conidios con un microscopio óptico (Motic B3 a 10× y 100×). Las fotografías y mediciones de las estructuras microscópicas se tomaron con el software Motic digital Microscope DMB3223 (Motic China Group Co., Ltd., 2001-2004). El número de mediciones de cada estructura se obtuvo de entre 15 y 20 muestras, las cuales se expresaron de la mínima a la máxima. Para la identificación de especies de Cordyceps s. $l$. se consultaron los trabajos de Kobayasi (1941, 1982); Kobayasi y Shimizu (1978); Mains (1951a, 1958); Pérez-Silva (1977, 1978); Petch (1933); Rubio-Bustos, Guzmán-Dávalos y Navarrete-Heredia (1999); Shrestha et al. (2014) y Yokoyama, Yamagishi y Hara (2005). Los insectos hospederos se identificaron hasta el nivel taxonómico posible con los trabajos de Alcázar-Ruiz, Morón-Ríos y Morón (2003); Bitar y Morón (2014); Conabio (2014); Deloya y Ratcliffe (1988); Lawrence y Newton (1995); Lemaire y Wolfe (1988). Todos los ejemplares fueron catalogados y depositados en el Acervo Micológico del Centro de Investigación en Recursos Bióticos (AMCIRB) de la Universidad Autónoma del Estado de México.

\section{Descripción}

\section{Familia Cordycipitaceae}

Cordyceps militaris (L.) Fr., Observer Mycology 2: 317. 1818 (fig. 1)

Estromas solitarios o gregarios, cilíndricos a clavados de $2-8 \mathrm{~cm}$ de longitud. Parte fértil cilíndrica a clavada, de $1.8-3 \mathrm{~cm}$ de largo $\times 5-8 \mathrm{~mm}$ de diámetro, color anaranjado (FFA500) a amarillo (FBE602). Estípite cilíndrico, liso, carnoso, de $2-5 \mathrm{~cm}$ de largo y 1-5 mm de ancho, color anaranjado claro (F6C204) a 

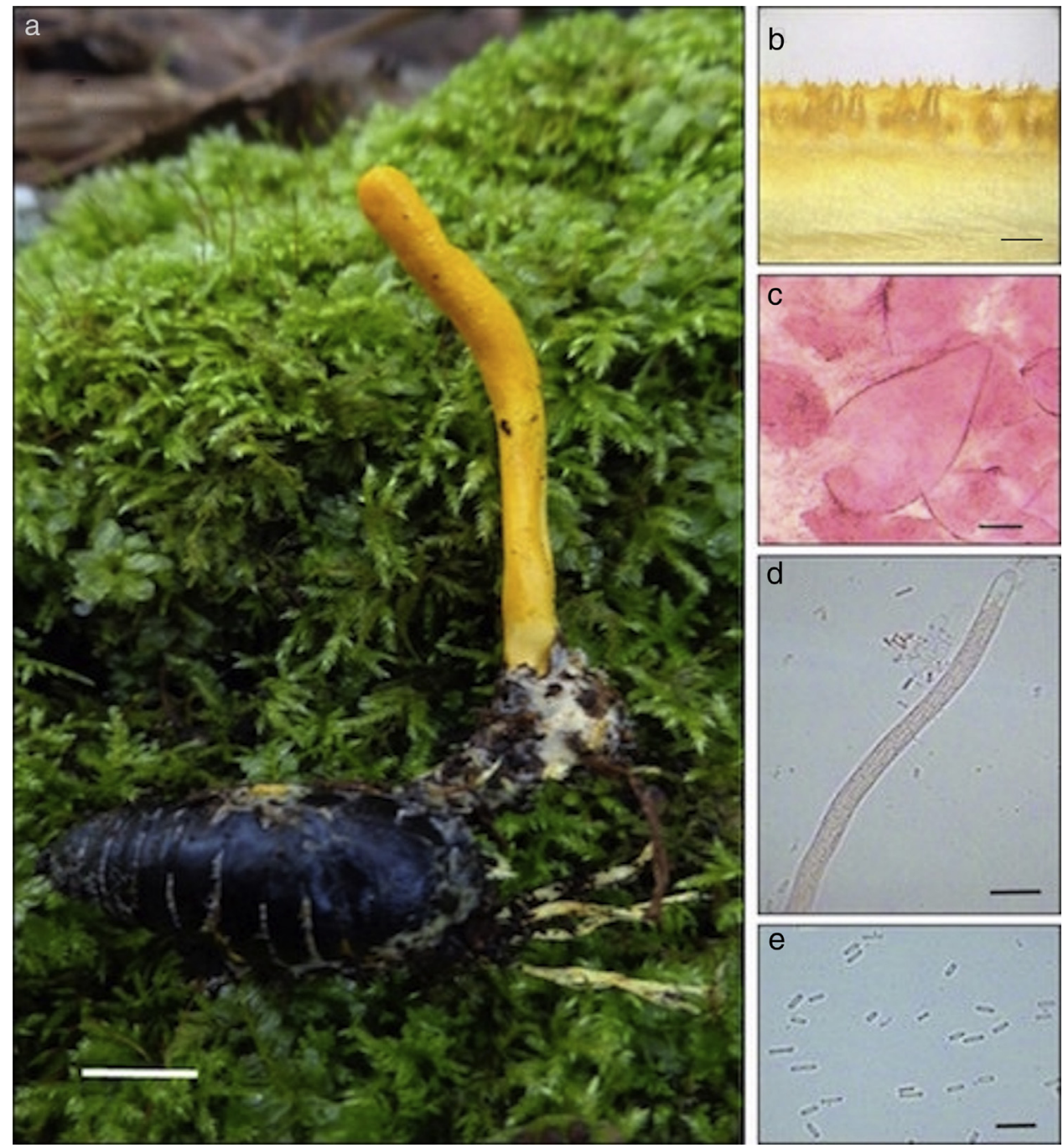

Figura 1. Cordyceps militaris: a) estroma emergiendo de una pupa de Paradirphia lasiocampina (lepidóptero); b) posición de los peritecios en el estroma; c) peritecios; d) asca con ascosporas multiseptadas, y e) ascosporas fragmentadas. Escalas: $1 \mathrm{~cm}$ (a), $0.5 \mathrm{~mm}$ (b), $100 \mu \mathrm{m}$ (c) y $10 \mu \mathrm{m}(\mathrm{d}-\mathrm{e})$.

amarillo (FBE602). Peritecios ovoides, completamente inmersos en el estroma, en ángulo recto, de 580-670 × 315-325 $\mu \mathrm{m}$ $(\mathrm{n}=20)$. Ascas cilíndricas y filamentosas, de 3-6 $\mu \mathrm{m}$ de ancho $(n=20)$. Ascosporas hialinas, filiformes, multiseptadas que se fragmentan en porciones de 3.5-4.2 × 1.4-2 $\mu \mathrm{m}(\mathrm{n}=20)$. Hospedero: pupas y larvas de Paradirphia lasiocampina (R. Felder et Rogenhofer) (Lepidoptera: Saturniidae) enterradas en el suelo. Hábitat: bosque de Quercus L.-Pinus L.; vegetación secundaria arbustiva de bosque de Quercus.

\section{Resumen taxonómico}

Estado de México: municipio Ocuilan, La Haciendita, $18^{\circ} 58^{\prime} 44^{\prime \prime}$ N, 99 25’01” O, 11-sep-2013, J.C. Pérez-Villamares (AMCIRB 29, 32); 23-oct-2013, J.C. Pérez-Villamares (AMCIRB 61, 62, 63, 64, 65, 67, 68, 69); 27-ago-2014, J.C. Pérez-Villamares (AMCIRB 135, 136, 137, 139); 08-oct-2014, J.C. Pérez-Villamares (AMCIRB 211, 212, 213, 214, 215, 216, 217, 218, 219, 221, 222, 223). Municipio Tenancingo, Santo Desierto El Carmen, $18^{\circ} 55^{\prime} 01$ ” N, 99³3’31” O, 07-ago-2013,
J.C. Pérez-Villamares (AMCIRB 1, 2, 3, 4, 5, 6, 7, 8, 9, 10, 11, 13, 14); 09-oct-2013, J.C. Pérez-Villamares (AMCIRB 41, 42, 43, 44, 45, 46, 47, 48, 49, 52); 03-jul-2014, J.C. Pérez-Villamares (AMCIRB 69, 73, 75); 13-ago-2014, J.C. Pérez-Villamares (AMCIRB 101, 102, 103, 104, 105, 106, 107, 108, 109, 110, $111,112,113,114,116,117,118,119,120,121,122,123$, 124, 125); 18-sep-2014, J.C. Pérez-Villamares (AMCIRB 181, 182, 183, 184, 185, 186, 187, 188, 189, 190, 191, 192, 193, 194, 195, 196, 197, 198, 199, 200, 201, 202, 203). Municipio Villa Guerrero, San Miguel, 1859’38’ N, 99³9'59’ O, 04sep-2013, J.C. Pérez-Villamares (AMCIRB 16, 17, 18, 19, 20, 21, 22, 23, 24, 25, 26, 27); 09-oct-2013, J.C. Pérez-Villamares (AMCIRB 53, 54, 55, 56, 57, 58, 59, 60); 31-jul-2014, J.C. Pérez-Villamares (AMCIRB 97, 98); 13-ago-2014, J.C. PérezVillamares (AMCIRB 115); 03-sep-2014, J.C. Pérez-Villamares (AMCIRB 143, 144, 145, 146, 147, 148, 149, 150, 151, 152, $153,154,155,156,157,158,159,161,162,163,164,165,166$, 168); 16-oct-2014, J.C. Pérez-Villamares (AMCIRB 224, 225 , 226, 227, 228, 229, 230, 231, 232, 233, 234, 235, 236, 237, 238, 239, 240, 242, 243, 244, 245). 


\section{Comentarios taxonómicos}

Cordyceps militaris presenta amplia distribución en México y se ha recolectado sobre larvas y pupas de lepidópteros nocturnos de las familias Geometridae, Noctuidae y Sphingidae en diferentes tipos de bosques como: bosque mesófilo de montaña, bosques de Pinus-Quercus, bosque tropical subcaducifolio, bosque tropical perennifolio, bosques de Abies Mill, Tsuga Carrière y Picea Link con vegetación decidua (Rubio-Bustos et al., 1999) y solo hay un reporte de Cordyceps militaris sobre coleópteros adultos de una selva baja caducifolia (Castro, Acosta-Urdapilleta, Valenzuela-Garza y Burgos-Solorio, 2012). Nikoh y Fukatsu (2000) comentan que algunas especies de Cordyceps s. l. pueden tener como hospedero una sola especie y otras presentan una amplia variedad de hospederos de un grupo taxonómico particular. A C.militaris se le puede encontrar en pupas de diferentes lepidópteros nocturnos, y algunos autores argumentan que la adaptación de C. militaris a tener como hospederos a insectos de diferentes grupos taxonómicos se debe a su presencia en diversas regiones geográficas y zonas ecológicas (Kobayasi, 1941; Mains, 1958; Sung y Spatafora, 2004). En el presente estudio, C. militaris fue la especie con mayor número de registros, los cuales se recolectaron sobre pupas y en algunos casos sobre larvas de Paradirphia lasiocampina exclusivamente en los sitios donde la vegetación predominante es de Quercus-Pinus y en los cuales predominan grandes poblaciones de este lepidóptero. De acuerdo con Rubinoff y Sperling (2002), los encinos son hospederos primarios de las larvas de $P$. lasiocampina. Fuxa y Tanada (1987) afirman que los hongos entomopatógenos se presentan constantemente en las poblaciones de sus hospederos $\mathrm{y}$, a medida que estos incrementan sus poblaciones, también se incrementa la población de los hongos entomopatógenos, desarrollando epizootias que representan un mecanismo de control natural de las poblaciones de hospederos.

Especies morfológicamente similares a Cordyceps militaris son: Cordyceps cardinalis G. H. Sung et Spatafora y Cordyceps roseostromata Kobayasi et Shimizu (Kobayasi y Shimizu, 1983; Shrestha, Zhang, Zhang y Liu, 2012; Sung y Spatafora, 2004; Wang, Zhang, Hu, Chen y Qu, 2008); no obstante, la primera presenta estromas más pequeños $(6-59 \mathrm{~mm})$, de color rojo, sus hospederos son larvas de lepidópteros, presentan rizomorfos y sus peritecios son más pequeños $(230-540 \times 110-240 \mu \mathrm{m})$, y en el caso de la segunda, los estromas son aún más pequeños (1.2$5 \mathrm{~mm}$ ), de color rosa, la parte fértil es subglobosa a clavada, presenta rizomorfos, sus peritecios son superficiales y mucho más pequeños $(280-300 \times 140-160 \mu \mathrm{m})$.

Cordyceps scarabaeicola Kobayasi, Bulletin of the National Science Museum, Tokyo, Series B, 2: 137.1976 (fig. 2)

Estromas solitarios o subgregarios, subclavados, de $1.5-8 \mathrm{~cm}$ de longitud. Parte fértil cilíndrica a subclavada, de 0.5-3.5 cm de longitud $\times 2.5-6 \mathrm{~mm}$ de diámetro, color anaranjado (E98A0E) a amarillo pálido (F7CC67), presencia de ostiolos oscuros que le dan una textura irregular a la parte fértil, diferenciándose ligeramente del estípite, ya que ambas estructuras son casi idénticas en diámetro y coloración. Estípite cilíndrico y liso, de $0.8-3.5 \mathrm{~cm}$ de longitud $\times 2-4 \mathrm{~mm}$ de diámetro, anaranjado claro (FABA66) a amarillo pálido (F7CC67). Peritecios ovoides con una punta obtusa, semi-inmersos, de 590-630 × 280$380 \mu \mathrm{m}(\mathrm{n}=15)$. Ascas cilíndricas, de 5-6.4 $\mu \mathrm{m}$ de diámetro $(n=15)$. Ascosporas hialinas, multiseptadas que se fragmen$\tan$ en esporas individuales de 5.2-7.5 × 1.3-1.8 $\mu \mathrm{m}(\mathrm{n}=15)$. Hospederos: coleópteros adultos de la familia Scarabaeidae: Cotinis mutabilis Gory et Percheron (subfamilia Cetoniinae), 2 especies no identificadas del género Phyllophaga (subfamilia Melolonthinae) y Xyloryctes thestalus Bates (subfamilia Dynastinae). Todos los hospederos se encontraron entre la hojarasca. Hábitat: bosque de Quercus-Pinus; ecotono entre vegetación secundaria arbustiva de bosque de Juniperus L. y una zona agrícola; vegetación secundaria arbustiva de bosque de Quercus.

\section{Resumen taxonómico}

Estado de México: municipio Malinalco, carretera Malinalco-Tenancingo, 18 58'19’'N, 99²9’40” O, 08-jul2014, J.C. Pérez-Villamares (AMCIRB 80); 01-oct-2014, J.C. Pérez-Villamares (AMCIRB 206). Municipio Ocuilan, La Haciendita, 18 58'44” N, 99 25'01’ O, 11-sep-2013, J.C. Pérez-Villamares (AMCIRB 28); 15-jul-2014, J.C. PérezVillamares (AMCIRB 87, 88). Municipio Tenancingo, Santo Desierto El Carmen, 18 55’01’ N, 99॰33’31’ O, 09-oct-2013, J.C. Pérez-Villamares (AMCIRB 50).

\section{Comentarios taxonómicos}

Cordyceps scarabaeicola se describió originalmente de Nueva Guinea (Kobayasi y Shimizu, 1976) sobre un coleóptero scarabaeoideo adulto y se ha reportado en países asiáticos como China (Liang, Liu y Feng, 1993), Corea (Shrestha et al., 2014; Sung, 1996), Japón (Kobayasi y Shimizu, 1978) y Taiwán (Kobayasi y Shimizu, 1981). Los ejemplares de C. scarabaeicola aquí descritos representan los primeros registros para México y el nuevo mundo, demostrando que el clado Beauveria no está solo circunscrito para el sureste asiático. Esta especie se caracteriza por desarrollar estromas que emergen de la parte cefálica de coleópteros adultos de la familia Scarabaeidae, de color amarillo limón, la parte fértil casi no se diferencia del estípite. Microscópicamente presenta peritecios ovalados y semi-inmersos y con una punta obtusa, lo que concuerda con lo observado por Kobayasi y Shimizu $(1976,1978)$ y Shrestha et al., 2014). Recientemente con datos moleculares se demostró que C. scarabaeicola es la forma teleomórfica del anamorfo Beauveria sungii S. A. Rehner y R. A. Humber (Shrestha et al., 2014). Una especie semejante es Cordyceps brongniartii Shimazu, la cual se puede diferenciar de la aquí estudiada por los estromas un poco más largos y clavados, surcados en la parte basal y de color amarillo a anaranjado-blanquecinos, y se desarrolla sobre larvas de coleópteros de la familia Scarabaeidae (Shimazu, Mitsuhashi y Hashimoto, 1988), mientras que los estromas de C. scarabaeicola son un poco más pequeños, cilíndricos y de color amarillo limón (Kobayasi y Shimizu, 1976, 1978; Shrestha et al., 2014) a anaranjado (presente estudio) y se desarrolla sobre coleópteros adultos de Scarabaeidae 

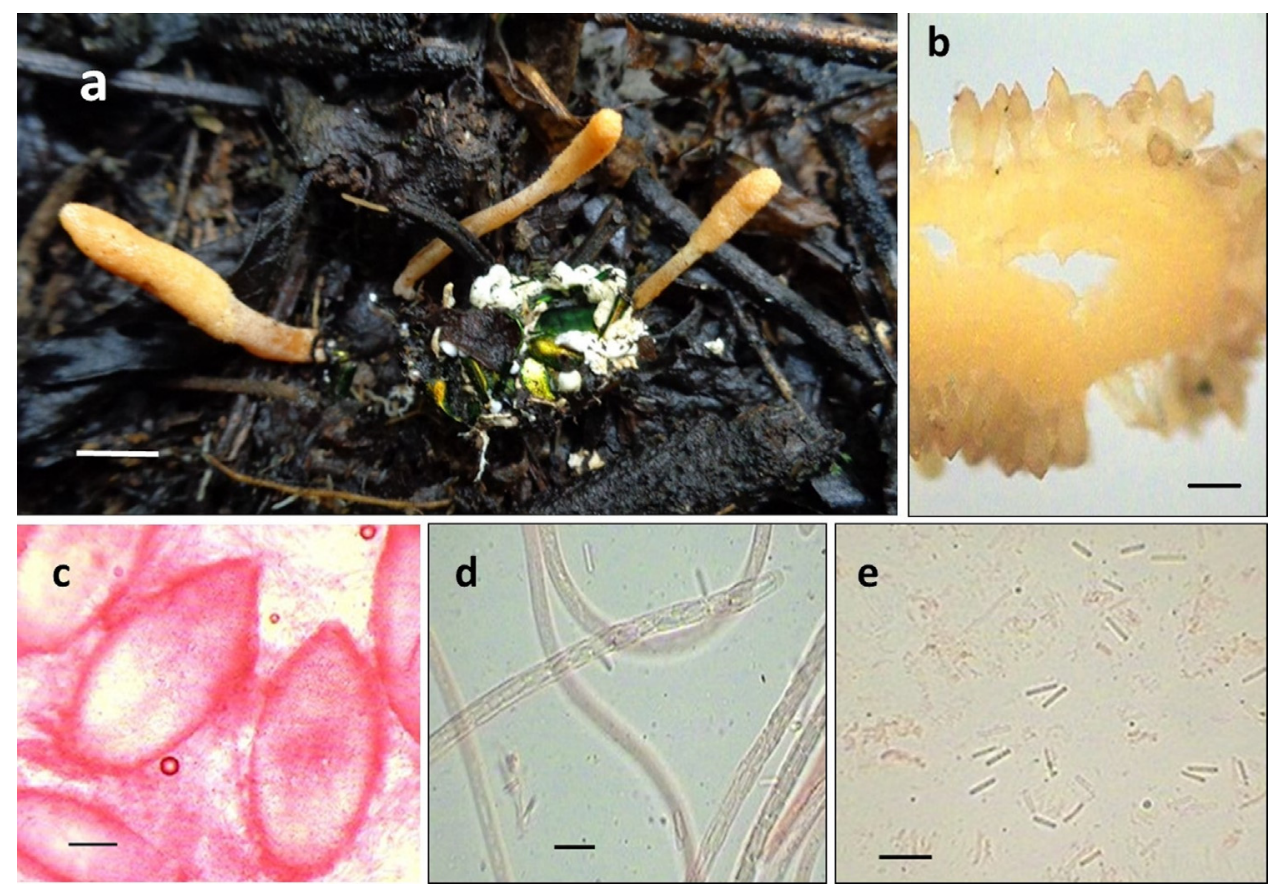

Figura 2. Cordyceps scarabaeicola: a) estromas emergiendo de un coleóptero adulto (Cotinis mutabilis); b) posición de los peritecios en el estroma; c) peritecios; d) asca con ascosporas multiseptadas, y e) ascosporas fragmentadas. Escalas: $1 \mathrm{~cm}(\mathrm{a}), 0.5 \mathrm{~mm}$ (b), $100 \mu \mathrm{m}$ (c) y $10 \mu \mathrm{m}(\mathrm{d}-\mathrm{e})$.
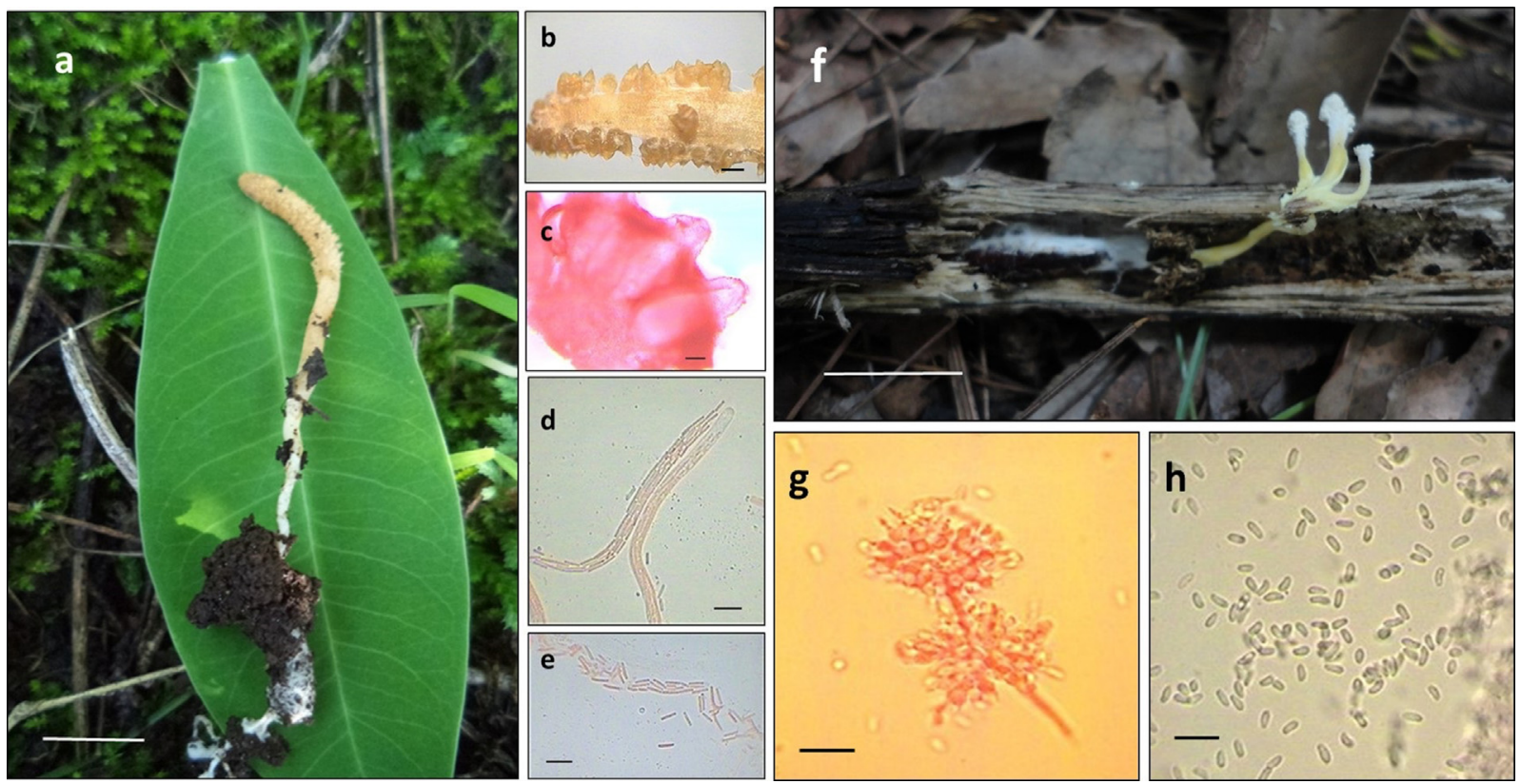

Figura 3. Cordyceps takaomontana. Teleomorfo (a-e): a) estroma emergiendo de su hospedero (pupa de lepidóptero); b) posición de los peritecios en el estroma; c) peritecios; d) asca con ascosporas multiseptadas, y e) ascosporas fragmentadas. Fase conidial (f-h): f) sinema emergiendo de una pupa (lepidóptero); g) conidióforo con fiálides, y h) conidios. Escalas: $1 \mathrm{~cm}$ (a y f), $0.5 \mathrm{~mm}$ (b), $100 \mu \mathrm{m}$ (c) y $10 \mu \mathrm{m}$ (d-h).

(Kobayasi y Shimizu, 1976, 1978; Shrestha et al., 2014; Sung et al., 2007).

Cordyceps takaomontana Yakush. et Kumaz., Science Reports of the Tokyo Bunrika Daigaku, Section B, 5: 108. 1941 (fig. 3)

Estromas solitarios o subgregarios, cilíndricos a clavados, de $1.5-6 \mathrm{~cm}$ de longitud. Parte fértil cilíndrica o clavada, de
$0.7-1.5 \mathrm{~cm}$ de longitud $\times 1.5-6 \mathrm{~mm}$ de diámetro, ostiolos superficiales, densos, mamiformes, anaranjados (E98A0E) a amarillo pálido (F0E68C). Estípite cilíndrico, liso o estriado longitudinalmente, simple o en algunos casos bifurcado, de $1-3.5 \mathrm{~cm}$ de longitud $\times 1-4 \mathrm{~mm}$ de diámetro, anaranjado claro (E3B673) a amarillo pálido (F0E68C). Peritecios ovoides, semi-inmersos o superficiales, de 450-580 $\times 220-250 \mu \mathrm{m}(\mathrm{n}=15)$. Ascas cilíndricas, de 4.4-5.6 $\mu \mathrm{m}$ de diámetro $(\mathrm{n}=15)$. Ascosporas cilíndricas, 
truncadas en ambos extremos, de 4.5-7.2 $\times$ 0.7-1.2 $\mu \mathrm{m}(\mathrm{n}=15)$. $\mathrm{Su}$ forma anamórfica presenta sinemas plumosos y ramificados, de 2 a $8 \mathrm{~cm}$ de longitud, de color blanco (FFFFFF). Estípite cilíndrico, de color amarillo limón (E6F78C). Conidióforos septados y ramificados. Fiálides piriformes con un cuello alargado. Conidios hialinos, faseoliformes, de 4-5 $\times 1.5-2 \mu \mathrm{m}(\mathrm{n}=15)$. Hospedero: pupas y larvas de lepidópteros enterradas en el suelo o entre ramas caídas. Hábitat: bosque de Quercus-Pinus; vegetación secundaria arbustiva de bosque de Quercus y el ecotono entre vegetación secundaria arbustiva de bosque de Juniperus y zona agrícola.

\section{Resumen taxonómico}

Estado de México: municipio Malinalco, carretera Malinalco-Tenancingo, $18^{\circ} 58^{\prime} 19^{\prime \prime} \mathrm{N}, \quad 99^{\circ} 29^{\prime} 40^{\prime \prime} \mathrm{O}, \quad 08$-jul2014, J.C. Pérez-Villamares (AMCIRB 76, 77, 78, 79, 81, 82, 83, 84, 85); 20-ago-2014, J.C. Pérez-Villamares (AMCIRB 126, 133, 134); 01-oct-2014, J.C. Pérez-Villamares (AMCIRB 204, 205). Municipio Ocuilan, La Haciendita, $18^{\circ} 58^{\prime} 44^{\prime \prime} \mathrm{N}$, 99 ${ }^{\circ}$ 25'01”'O, 15-jul-2014, J.C. Pérez-Villamares (AMCIRB 86, 89); 08-oct-2014, J.C. Pérez-Villamares (AMCIRB 210, 220). Municipio Tenancingo, Santo Desierto El Carmen, 18 55’01” N, 99³3’31” O, 03-jul-2014, J.C. Pérez-Villamares (AMCIRB 71, 72, 74). Municipio Tenango del Valle, San Pedro Tlanixco, 1903'45” N, 99 39'07” O, 11-sep-2014, J.C. Pérez-Villamares (AMCIRB 177, 178, 179, 180); 18-sep-2013, J.C. Pérez-Villamares (AMCIRB 33); 06-ago-2014, J.C. Pérez-Villamares (AMCIRB 100); Municipio Villa Guerrero, San Miguel, 18 ${ }^{\circ} 59^{\prime} 38^{\prime \prime}$ N, 99³9'59” O, 03-sep-2014, J.C. Pérez-Villamares (AMCIRB 160, 167); 16-oct-2014, J.C. Pérez-Villamares (AMCIRB 241).

\section{Comentarios taxonómicos}

Cordyceps takaomontana fue descrito originalmente en Japón (Kobayasi, 1941) y se ha reportado de otros países como China (Liang, Liu, Liu y Kang, 2003), Colombia (Sanjuan, 2014), el Congo (Moureau, 1961), Corea (Lee, Hwang y Yun, 2009) y Rusia (Koval, 1974). En México solamente se había reportado su fase conidial descrita como Paecilomyces tenuipes (Peck) Samson, de un bosque mesófilo de montaña al este de México, en el estado de Veracruz (López y García, 2002). En este estudio se reporta por primera vez para México al holomorfo, ampliando su distribución hacia el centro de México. De acuerdo con Kobayasi (1941), esta especie se caracteriza por desarrollar estromas cilíndricos, en ocasiones bifurcados y de color amarillo pálido, los cuales crecen sobre pupas de lepidópteros. Los peritecios pueden ser superficiales o subinmersos con ostiolos mamiformes que sobresalen en la parte fértil del estroma. De los materiales estudiados, las características macro y micro-morfológicas coinciden con las reportadas en la descripción original de la especie, en la que se menciona el desarrollo de estromas sobre pupas de lepidópteros. Koval (1974) cita a C. takaomontana también sobre larvas y pupas de lepidópteros de la familia Sphingidae; además del color amarillo pálido, describe ejemplares con una coloración más oscura, ocre pálido. Por otro lado, Sanjuan (2014) reporta que en ecosistemas con baja perturbación antrópica $C$. takaomontana se desarrolla únicamente sobre pupas de lepidópteros de la familia Noctuidae y de la tribu Ithomiinae Nymphalidae, mientras que en ecosistemas manejados por el hombre como un mariposario produce epizootias en larvas y pupas de diferentes especies de lepidópteros de diferentes subfamilias: Heliconinae, Hespiaridae, Papilionidae y Pieridae. Una especie muy similar a C.takaomontana es Cordyceps ochraceostromata Kobayasi et Shimizu, la cual se diferencia por la coloración ocre pálido o rojizo marrón de los estromas, mientras que en C. takaomontana son de color amarillo pálido; los peritecios en C. ochraceostromata están completamente inmersos en el tejido estromático (Kobayasi y Shimizu, 1980).

\section{Familia Ophiocordycipitaceae}

- Ophiocordyceps entomorrhiza (Dicks.) G.H. Sung, J.M. Sung, Hywel-Jones et Spatafora, Studies in Mycology, 57: 42. 2007 (fig. 4)

Estromas solitarios o subgregarios, capitados, de $3-8 \mathrm{~cm}$ de longitud. Parte fértil esférica, de $2-5 \times 3-5 \mathrm{~mm}$, rojizo pálido (EE8D61) a rojizo $(\mathrm{B} 2420 \mathrm{~F})$ de $4.5-5 \times 5-6 \mathrm{~mm}$, superficie muy rugosa por la presencia de ostiolos. Estípite cilíndrico, de $25-75 \times 1.5-2 \mathrm{~mm}$, pruinoso en la superficie, en algunos casos curvado, completamente blanco (FFFFFF) o color café claro arriba (FFEBCD) y café oscuro abajo (B95323) y con abundantes cordones miceliales adheridos al sustrato entre 10$30 \mathrm{~mm}$ de longitud y no más de $1 \mathrm{~mm}$ de diámetro y de color blanco (FFFAFA) a café claro (DEB887). Peritecios inmersos en el estroma, ovoides, de 800-900 $\times 300-350 \mu \mathrm{m}(\mathrm{n}=20)$. Ascas cilíndricas de 5.1-5.6 $\mu \mathrm{m}$ de diámetro $(\mathrm{n}=20)$. Ascosporas filiformes, multiseptadas, fragmentándose en segmentos individuales de $8.7-10.3 \times 1-2 \mu \mathrm{m}(\mathrm{n}=20)$. Hospedero: larvas de coleóptero enterradas en el suelo. Hábitat: vegetación secundaria arbustiva de bosque de Quercus.

\section{Resumen taxonómico}

Estado de México, municipio Villa Guerrero, San Miguel, $18^{\circ} 59^{\prime} 38^{\prime \prime}$ N, 99`39’59”' O, 31-jul-2014, J.C. Pérez-Villamares (AMCIRB 91, 92, 93, 94, 95, 96, 99); 03-sep-14, J.C. PérezVillamares (AMCIRB 169, 170, 171, 172, 173, 174 175, 176).

\section{Comentarios taxonómicos}

Ophiocordyceps entomorrhiza solamente se había reportado para Veracruz, en la región centro- este de México, en agroecosistemas cafetaleros (citado como Cordyceps entomorrhiza (Fr.) Link) (Pérez-Silva, 1978). Esta especie es muy cercana a Ophiocordyceps gracilis, con la cual ha sido frecuentemente confundida (Mains, 1951b; Petch, 1933; Rubio-Bustos et al., 1999). De acuerdo con Allard (1998), Eckblad (1967), Mains (1951b, 1958) y Zang y Kinjo (1998), O.gracilis tiene la parte fértil del estroma de color rojo, anaranjado u ocre, con numerosos ostiolos oscuros en la superficie; los peritecios son completamente inmersos, y el estípite es amarillo o amarillento. En contraste, O. entomorrhiza tiene la parte fértil del estroma de color vino, gris violáceo o marrón grisáceo, con la superficie verrugosa por la proyección de los peritecios; el estípite marrón pálido arriba y marrón oscuro abajo, además posee cordones 


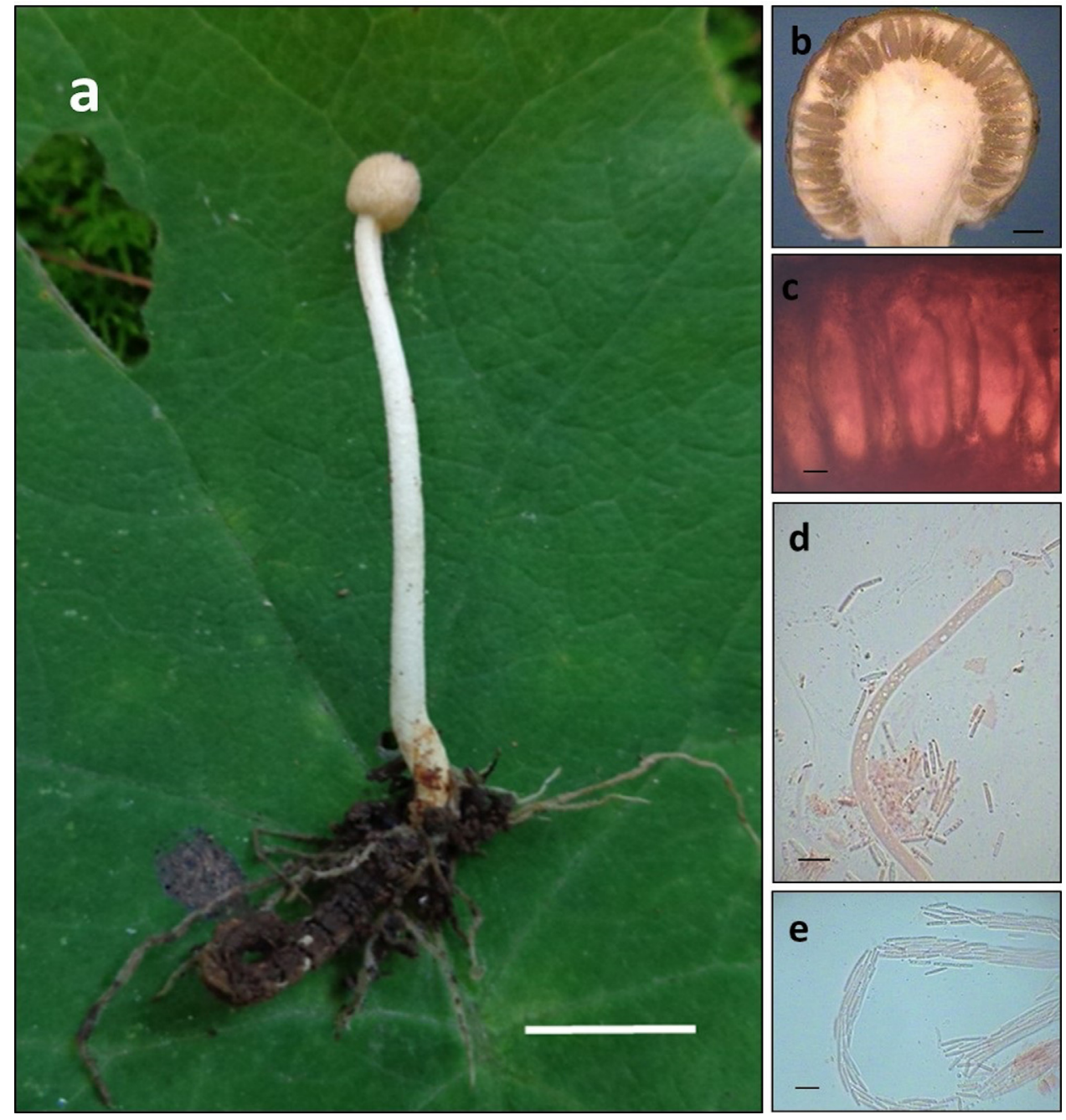

Figura 4. Ophiocordyceps entomorrhiza: a) estroma emergiendo de una larva de coleóptero; b) posición de los peritecios en el estroma; c) peritecios; d) asca, y e) ascosporas. Escalas: $1 \mathrm{~cm}$ (a), $0.5 \mathrm{~mm}$ (b), $100 \mu \mathrm{m}$ (c) y $10 \mu \mathrm{m}$ (d-e).

miceliales, similares a las raíces de plantas vasculares, de los cuales se origina su nombre. Los hospederos de $O$. gracilis son larvas de Lepidoptera y probablemente restringida a este orden, mientras $O$. entomorrhiza es probablemente específico de larvas de coleópteros. La coloración rojiza de los estromas analizados en este estudio difieren en la coloración vino, gris violáceo o marrón grisáceo citada por otros autores (Allard, 1998; Eckblad, 1967; Mains, 1951b, 1958; Zang y Kinjo, 1998). Por otro lado, los ejemplares descritos por Pérez-Silva (1978) en Veracruz son similares en coloración tanto de la parte fértil como del estípite. Al respecto, Guzmán et al. (2001) argumentan que aunque la coloración de los estromas descritos por Pérez-Silva (1978) es muy similar a la que presenta $O$. gracilis, la descripción corresponde a $O$. entomorrhiza.

Ophiocordyceps gracilioides (Kobayasi) G.H. Sung, J.M. Sung, Hywel-Jones et Spatafora, Studies in Mycology, 57: 43. 2007 (fig. 5)

Estromas capitados, solitarios o subgregarios, que emergen de la cabeza del hospedero, de $1.5-2 \mathrm{~cm}$ de longitud. Parte fértil esférica, de $2-3 \mathrm{~mm}$ de longitud $\times 3.5-4 \mathrm{~mm}$ de diámetro, de color rojizo (B03609). Estípite cilíndrico, de 17-18 mm de largo $\times 1.5-2 \mathrm{~mm}$ de ancho, color crema (FBDD92) con tonos anaranjado claro (EA721D). Peritecios completamente inmersos y elongados, de $800-890 \times 220-280 \mu \mathrm{m}(\mathrm{n}=15)$. Ascas cilíndricas, de 5.8-7 $\mu \mathrm{m}$ de diámetro $(\mathrm{n}=15)$. Ascosporas cilíndricas, hialinas, que se rompen en fragmentos individuales, de 7.5-8.7 $\times 1.3-1.9 \mu \mathrm{m}(\mathrm{n}=15)$. Hospedero: larvas de coleóptero enterradas en el suelo. Hábitat: ecotono entre vegetación secundaria arbustiva de bosque de Juniperus y una zona agrícola.

\section{Resumen taxonómico}

Estado de México: municipio Malinalco, carretera Malinalco-Tenancingo, $18^{\circ} 58^{\prime} 19^{\prime \prime} \mathrm{N}, \quad 99^{\circ} 29^{\prime} 40^{\prime \prime} \mathrm{O}, \quad 25-$ sep-2013, J.C. Pérez-Villamares (AMCIRB 36, 37, 38, 39, 40); 20-ago-2014, J.C. Pérez-Villamares (AMCIRB 127).

\section{Comentarios taxonómicos}

Ophiocordyceps gracilioides se describió originalmente de Japón sobre larvas de coleóptero (Kobayasi, 1941) y se ha reportado de China (Fan, Li, Chen y Li, 2001, Li, Fan, Huang y Li, 2002) y Colombia (Sanjuan, 2014). Recientemente, 

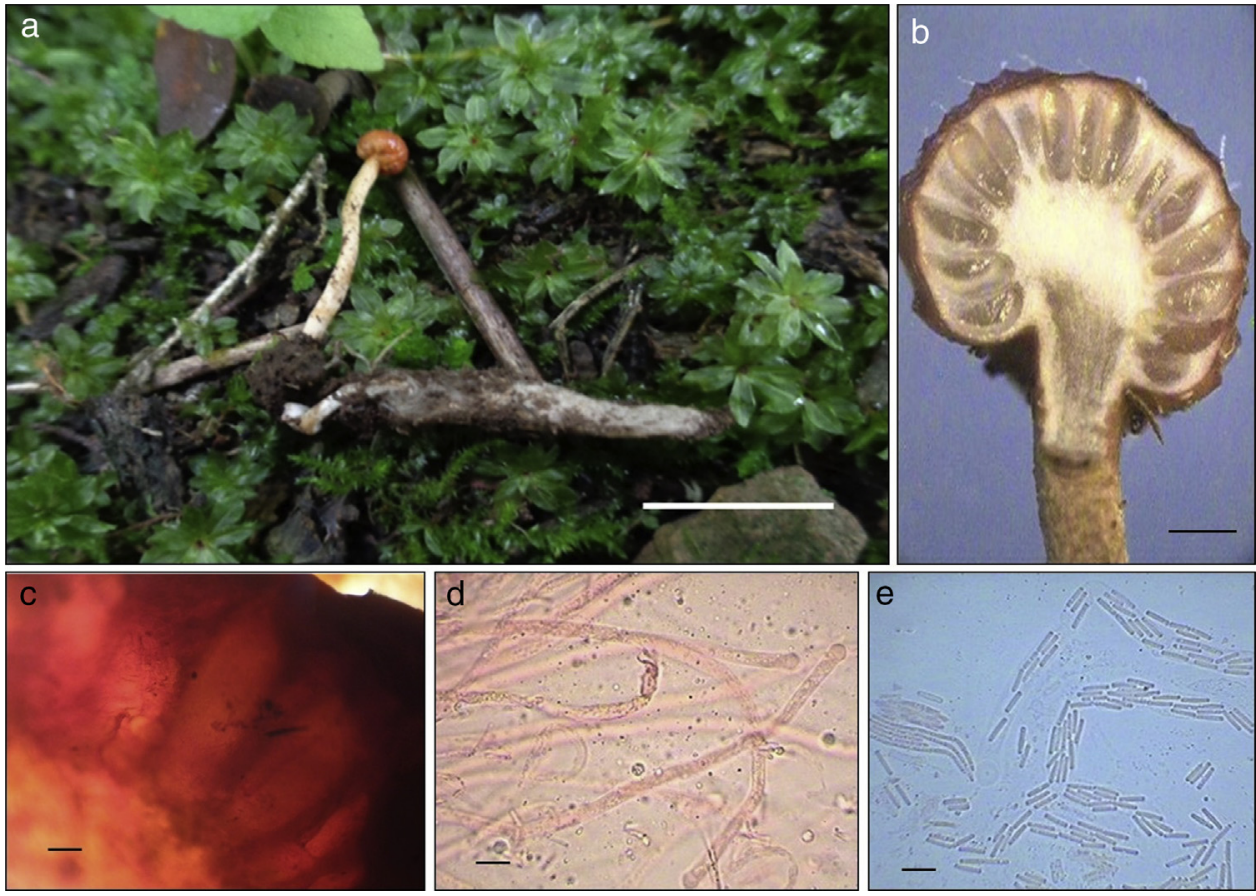

Figura 5. Ophiocordyceps gracilioides: a) estroma emergiendo de una larva de coleóptero; b) posición de los peritecios en el estroma; c) peritecios; d) asca, y e) ascosporas. Escalas: $1 \mathrm{~cm}(\mathrm{a}), 1 \mathrm{~mm}(\mathrm{~b}), 100 \mu \mathrm{m}$ (c) y $10 \mu \mathrm{m}$ (d-e).

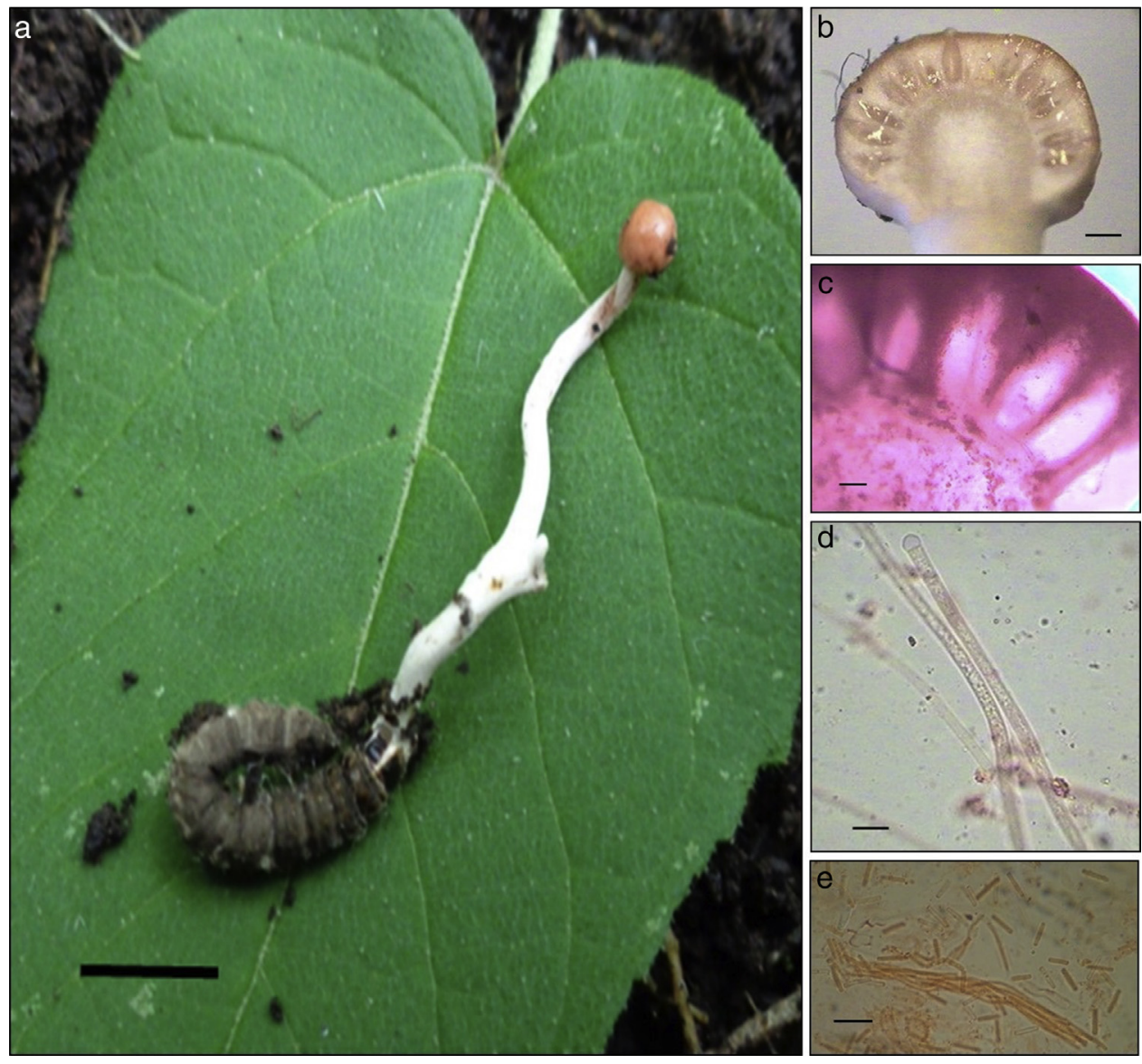

Figura 6. Ophiocordyceps gracilis: a) estroma emergiendo de una larva de lepidóptero; b) posición de los peritecios en el estroma; c) peritecios; d) asca, y e) ascosporas. Escalas: $1 \mathrm{~cm}(\mathrm{a}), 0.5 \mathrm{~mm}$ (b) $100 \mu \mathrm{m}$ (c) y $10 \mu \mathrm{m}$ (d-e). 

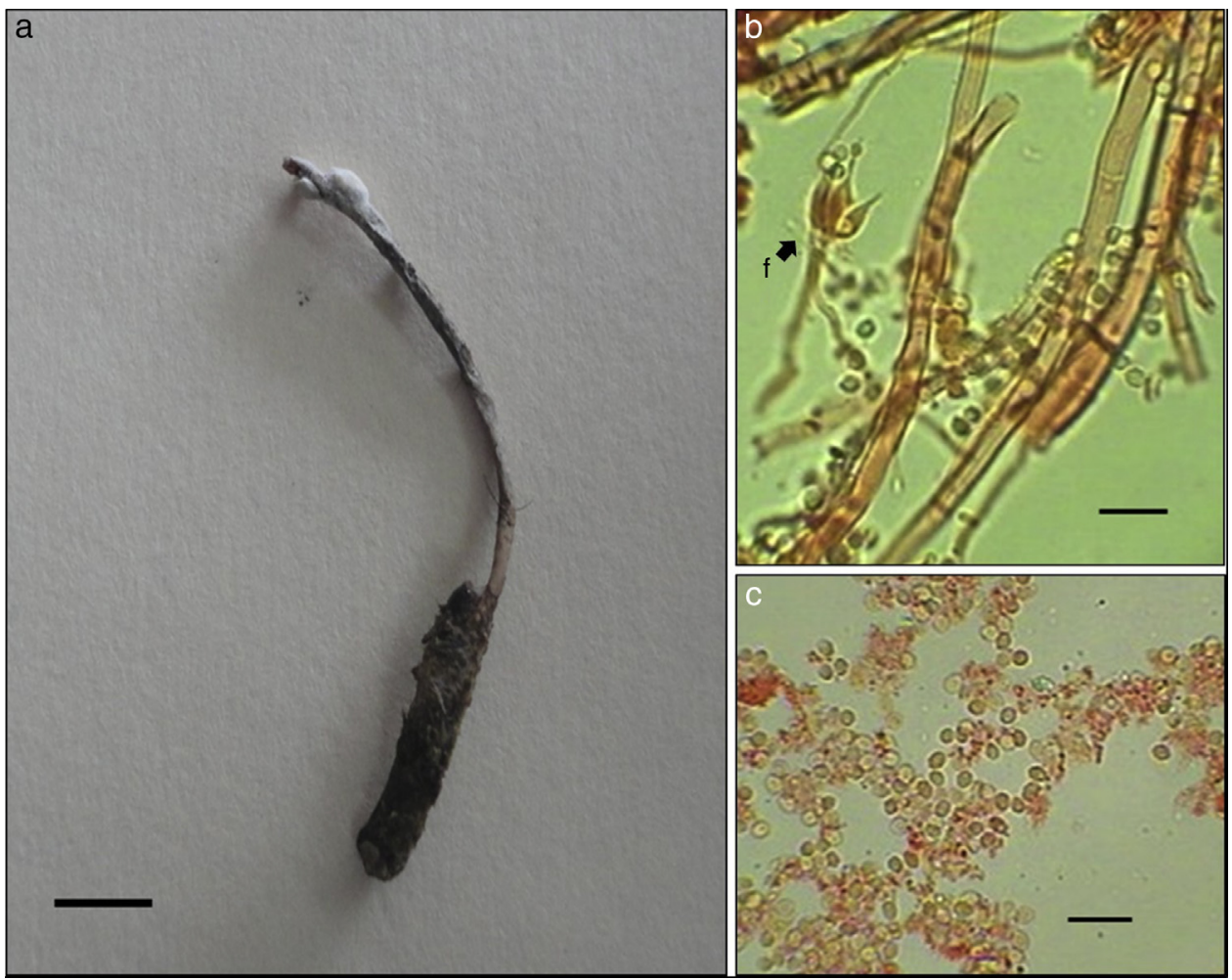

Figura 7. Ophiocordyceps stylophora: a) sinema emergiendo del tórax de una larva de coleóptero; b) fiálides con conidios, y c) conidios. Escalas: $1 \mathrm{~cm}$ (a) y $10 \mu \mathrm{m}$ (b y c).

Castro et al. (2012) registraron por primera vez a $O$. gracilioides sobre larvas de coleópteros de una selva baja caducifolia del centro de México. Los ejemplares reportados en este estudio representan el segundo reporte de la especie del centro de México y sus características coinciden con las descripciones de Fan et al. (2001) y Kobayasi (1941), existiendo solo una ligera diferencia en cuanto al tamaño de los estromas que cita el segundo autor son más largos. De acuerdo con Kobayasi (1941), entre las especies similares a $O$. gracilioides se encuentran O. gracilis y O. glaziovii (Henn.) G.H. Sung, J.M. Sung et al., 2007. $O$. gracilis se diferencia de $O$. gracilioides porque sus estromas y peritecios son más pequeños, la parte fértil es de color castaño y sus hospederos son larvas de lepidóptero. Por su parte, O. glaziovii desarrolla estromas ligeramente más pequeños a la especie aquí descrita y la coloración de su parte fértil es color marrón a color café (Kobayasi, 1941).

- Ophiocordyceps gracilis (Grev.) G.H. Sung, J.M. Sung, Hywel-Jones et Spatafora, Studies in Mycology, 57: 43. 2007 (fig. 6)

Estromas capitados, solitarios o en pares, erectos o curvados, de 2-3.5 cm de largo, emergiendo del tórax del hospedero. Parte fértil esférica, de $2-5 \times 2-4 \mathrm{~mm}$, de color salmón claro (FD8962) a rojizo (AA3F1B). Estípite cilíndrico, recto o curvado, de $15-35 \mathrm{~mm}$ de longitud $\times 1-2 \mathrm{~mm}$ de diámetro, color blanco (FFFFFF) a crema (FBDD92) con tonos anaranjado claro (EA721D). Peritecios ovoides y elongados, completamente inmersos, de 640-660 × 220-250 $\mu \mathrm{m}(\mathrm{n}=15)$. Ascas cilíndricas de 4.2-5.5 $\mu \mathrm{m}$ de diámetro $(\mathrm{n}=20)$ y rostradas. Ascosporas hialinas, filiformes, multiseptadas, las cuales se rompen en fragmentos de 6-10 $\mu \mathrm{m} \times 1-1.8 \mu \mathrm{m}(\mathrm{n}=15)$. Hospedero: larvas de lepidóptero. Hábitat: ecotono entre vegetación secundaria de bosque de Juniperus y una zona agrícola.

\section{Resumen taxonómico}

Estado de México: municipio Malinalco, carretera Malinalco-Tenancingo, $18^{\circ} 58^{\prime} 19^{\prime \prime} \mathrm{N}, 9^{\circ} 29^{\prime} 40$ ' O, 25-sep2013, J.C. Pérez-Villamares (AMCIRB 34, 35); 20-ago-2014, J.C. Pérez-Villamares (AMCIRB 128, 129, 130, 131, 132).

\section{Comentarios taxonómicos}

Ophiocordyceps gracilis solamente se había reportado de la región centro-oeste de México, en bosque tropical caducifolio y vegetación inducida (Rubio-Bustos et al., 1999). Posteriormente, Guzmán et al. (2001) hacen una revisión de los ejemplares reportados por Rubio-Bustos et al. (1999) como O. entomorrhiza sobre larvas de lepidópteros de la familia Noctuidae de un bosque mesófilo de montaña la región Este de México, concluyendo que en realidad corresponden a $O$. gracilis y no a $O$. entomorrhiza. El material tratado en este estudio coincide con la descripción citada por Mains (1958) y se amplía su distribución ahora para el centro de México. Las especies más similares a $O$. gracilis son $O$. entomorrhiza y $O$. gracilioides, las cuales se discutieron anteriormente.

- Ophiocordyceps stylophora (Berk. et Broome) G.H. Sung, J.M. Sung, Hywel-Jones et Spatafora, Studies in Mycology, 57: 47. 2007 (fig. 7) 
Para esta especie solo se describe la fase conidial, la cual presenta sinemas solitarios, cilíndricos, emergiendo del tórax del hospedero, de $3 \mathrm{~cm}$ de largo. Parte conidiógena de $10 \mathrm{~mm}$ de longitud $\times 2 \mathrm{~mm}$ de diámetro, de color blanco (FFFAFA); conidióforos ramificados y septados, de 4.4-5.4 $\mu \mathrm{m}$ de diámetro $(n=15)$. Fiálides hialinas elipsoides con la base muy ancha $\mathrm{y}$ un cuello muy delgado, de 10.8-11.5 $\mu \mathrm{m}(\mathrm{n}=15)$; conidios ovoides hialinos cubiertos con una sustancia mucilaginosa, de 3.1-3.9 $\times 2.3-2.5 \mu \mathrm{m}$ de diámetro $(\mathrm{n}=20)$. Estípite cilíndrico, de $2 \mathrm{~cm}$ de longitud $\times 1-1.5 \mathrm{~mm}$ de diámetro, de color grisáceo en la parte superior (242321) y en la base rosa pálido. Hospedero: larvas de coleóptero. Hábitat: bosque de Quercus-Pinus

\section{Resumen taxonómico}

Estado de México: municipio Tenancingo, Santo Desierto El Carmen, 18 55’01”N, 99 33’31”'O, 07-ago-2013, J.C. Pérez-Villamares (AMCIRB 12, 15); 09-oct-2013, J.C. PérezVillamares (AMCIRB 51).

\section{Comentarios taxonómicos}

Ophiocordyceps stylophora es una especie rara vez registrada en diferentes regiones del mundo (Chachula, Voncina y Kozik, 2011). El teleomorfo fue descrito por primera vez por Berkeley (1857) (citado como Cordyceps stylophora) en Carolina del Sur en Estados Unidos, país donde comúnmente se han recolectado (Kobayasi, 1941; MacMillan, 1898; Mains, 1958); también se ha reportado en otras localidades del mundo: China (Kobayasi, 1941), Japón (Ban, Sakane, Toyama y Nakagiri, 2009), Polonia (Chachula et al., 2011) y Suecia (Nordén, Appelqvist y Olausson, 2002). Mains (1951a) describió por primera vez la fase conidial (como Hirsutella stylophora) sobre larvas de coleóptero en los estados de Michigan, Nueva York y Tennessee, Estados Unidos, y se ha reportado en Nueva Zelanda sobre larvas de Costelytra zealandica (White) (Coleoptera: Scarabaeidae) (Glare, 1992) y Portugal (Moniz, Cabral, Tomaz y Basto, 1999). Pérez-Silva (1978) citó por primera vez para México al holomorfo (como Cordyceps stylophora al teleomorfo e Hirsutella stylophora a la fase conidial) sobre un nuevo hospedero, el cual corresponde a una avispa. Sin embargo, la autora argumenta que las características morfológicas coinciden con la descripción de la especie. Los ejemplares descritos en este estudio en su fase conidial concuerdan claramente con la descripción de Mains (1951a) con ejemplares de Norteamérica y con la descripción de Glare (1992) con ejemplares de Nueva Zelanda. De acuerdo con Mains $(1941,1958)$, la fase conidial de O. stylophora es comúnmente más recolectada que al teleomorfo debido que los estromas se desarrollan a finales del verano y maduran hasta la primavera siguiente.

\section{Agradecimientos}

$\mathrm{Al}$ Conacyt por la beca otorgada al primer autor para la realización del presente trabajo. A la Universidad Autónoma del Estado de México a través de la Secretaría de Investigación y Estudios Avanzados por el financiamiento de la investigación (SyEA 3687/2014/CIA).

\section{Referencias}

Alcázar-Ruiz, J. A., Morón-Ríos, A. y Morón, M. A. (2003). Fauna de Coleoptera Melolonthidae de Villa las Rosas, Chiapas, México. Acta Zoológica Mexicana (nueva serie), 88, 59-86.

Allard, C. (1998). Rencontres avec Cordyceps gracilis Mont. et Durieu: Le mystère des rendez-vous mycologiques. Bulletin de la Société Mycologique de France, 114, 29-33.

Ban, S., Sakane, T., Toyama, K. y Nakagiri, A. (2009). Teleomorph-anamorph relationships and reclassification of Cordyceps cuboidea and its allied species. Mycoscience, 50, 261-272.

Berkeley, M. J. (1857). On some entomogenous Sphaerie. Journal of the Proceedings of Linnean Society. Botany, 1, 157-159.

Bitar, A. y Morón, M. A. (2014). Revisión y análisis filogenético del género Xyloryctes (Coleoptera: Melolonthidae: Dynastinae: Oryctini). Revista Mexicana de Biodiversidad, 85, 753-796.

Castro, B. D., Acosta-Urdapilleta, M. L., Valenzuela-Garza, R. y BurgosSolorio, A. (2012). Hongos entomopatógenos del género Cordyceps s.l (Fungi: Ascomycota) en el estado de Morelos. En A. Equihua, E. G. Estrada, J. A. Soto, M. P. Chaires, y G. Durán (Eds.), Entomología mexicana (pp. 273-276). México D.F.: Sociedad Mexicana de Entomología A.C.

Chachula, P., Voncina, G. y Kozik, J. (2011). Ophiocordyceps stylophora (Ascomycota Hypocreales), new species for Poland. Polish Botanical Journal, 56, 321-326.

Conabio (Comisión Nacional para el Conocimiento y Uso de la Biodiversidad) (2014). Paradirphia lasiocampina [consultado 28 Oct 2014]. Disponible en: http://conabio.inaturalist.org/taxa/257241-Paradirphia-lasiocampina

Deloya, C. y Ratcliffe, B. C. (1988). Las especies de Cotinis Burmeister en México (Coleoptera: Melolonthidae: Cetoniinae). Acta Zoológica Mexicana (nueva serie), 28, 1-52.

Eckblad, F. E. (1967). The genus Cordyceps in Norway. Nytt Magasin for Botanik, 14, 68-76.

Fan, M. Z., Li, C. R., Chen, Y. Y. y Li, Z. Z. (2001). Cordyceps gracilioides, a new record for China. Mycosystema, 20, 273-274.

Frutis, I., Chio, R. E. y Estrada-Torres, A. (1985). Nuevos registros de macromicetos del Estado de México. Revista Mexicana de Micología, 1, 285-300.

Fuxa, J. R. y Tanada, Y. (1987). Epizootiology of insect diseases. New York: Wiley-Interscience.

Glare, T. R. (1992). Hirsutella stylophora Mains, a pathogen of Costelytra zealandica. New Zealand Entomologist, 15, 29-32.

Guzmán, G., Morón, M. A., Ramírez-Guillén, F. y Wolf, J. H. D. (2001). Entomogenous Cordyceps and related genera from Mexico with discussions on their hosts and new records. Mycotaxon, 78, 115-125.

Heim, R. y Wasson, R. G. (1958). Les champignons hallucinogènes du Mexique, études 682 ethnologiques, taxinomiques, biologiques physiologiques et chimiques. París: Muséum National d'Histoire Naturelle.

Hibbett, D. S. y Taylor, J. W. (2013). Fungal systematics: Is a new age of enlightenment at hand? Nature Reviews Microbiology, 11, 129-133.

HTML Color Codes [consultado 9 Abr 2016]. Disponible en: http://html-color-codes.info/

Index Fungorum (2015). Authors of fungal names [consultado 10 Feb 2015] Disponible en: http://www.indexfungorum.org/names/names.asp

Kepler, R. M., Humber, R. A., Bischoff, J. F. y Rehner, S. A. (2014). Clarification of generic and species boundaries for Metarhizium and related fungi through multigene phylogenetics. Mycologia, 106, 811-829.

Kobayasi, Y. (1941). The genus Cordyceps and its allies. Science Reports of the Tokyo Bunrika Daigaku (Section B 84), 5, 53-260.

Kobayasi, Y. (1982). Keys to the taxa of the genera Cordyceps and Torrubiella. Transactions of the Mycological Society of Japan, 23, 329-364.

Kobayasi, Y. y Shimizu, D. (1976). The genus Cordyceps and its allies from New Guinea. Bulletin of National Science Museum Tokyo Series B (Botany), 2, 133-151.

Kobayasi, Y. y Shimizu, D. (1978). Cordyceps species from Japan. Bulletin of the National Science Museum Tokyo Series B (Botany), 4, 43-63.

Kobayasi, Y. y Shimizu, D. (1980). Cordyceps species from Japan 3. Bulletin of the National Science Museum Tokyo Series B (Botany), 6, 125-145. 
Kobayasi, Y. y Shimizu, D. (1981). The genus Cordyceps and its allies from Taiwan (Formosa). Bulletin of the National Science Museum Tokyo Series B (Botany), 7, 113-122.

Kobayasi, Y. y Shimizu, D. (1983). Cordyceps from Japan 6. Bulletin of the National Science Museum Tokyo Series B (Botany), 9, 1-21.

Koval, E. Z. (1974). Opredelitel Entomofilnych Gribov SSSR. Rusia: CCCP Kiev.

Lawrence, J. F. y Newton, A. F., Jr. (1995). Families and subfamilies of Coleoptera (with selected genera, notes, references and data on family-group names). En J. Pakaluk y S. A. Slipinski (Eds.), Biology, phylogeny, and classification of Coleoptera: papers celebrating the 80th birthday of Roy A. Crowson (pp. 779-1006). Varsovia: Muzeum I Instytut Zoologii PAN.

Lee, S. H., Hwang, H. S. y Yun, J. W. (2009). Production of polysaccharides by submerged mycelial culture of entomopathogenic fungus Cordyceps takaomontana and their apoptotic effects on human neuroblastoma cells. Korean Journal of Chemical Engineering, 26, 1075-1083.

Lemaire, C. y Wolfe, K. L. (1988). Three new species of Paradirphia (Saturniidae: Hemileucinae) from Mexico and Central America with notes on the immature stages. Journal of Research on the Lepidoptera, 27, 197-212.

Li, C. R., Fan, M. Z., Huang, B. y Li, Z. Z. (2002). The genus Cordyceps and its allies from Anhui II. Mycosystema, 21, 167-171.

Liang, Z. Q., Liu, A. Y. y Feng, D. M. (1993). Some entomogeous fungi from Fanjing mountain preserve in China. Acta Mycologica Sinica, 12, 110-117.

Liang, Z. Q., Liu, A. Y., Liu, M. H. y Kang, J. C. (2003). The genus Cordyceps and its allies from the Kuankuoshui Reserve in Guizhou III. Fungal Diversity, 14, 95-101.

López, A. y García, J. (2002). Paecilomyces tenuipes. Funga Veracruzana, 76 , $1-4$.

MacMillan, C. (1898). Cordyceps stylophora Berk. et Br. in Minesota. Bulletin of the Torrey Botanical Club, 25, 583.

Mains, E. B. (1941). Cordyceps stylophora and Cordyceps ravenelii. Mycologia, 33, 611-617.

Mains, E. B. (1951a). Entomogenous species of Hirsutella stylophora, Tilachildium and Synnematium. Mycologia, 43, 691-718.

Mains, E. B. (1951b). Notes concerning entomogenous fungi. Bulletin of the Torrey Botanical Club, 78, 122-133.

Mains, E. B. (1958). North American entomogenous species of Cordyceps. Mycologia, 50, 169-222.

Moniz, M. F., Cabral, M. T., Tomaz, I. L. y Basto, M. S. (1999). On the appearance of a mycosis in Phoracantha semipunctata (Fab.) larva. Silva Lusitana, $7,49-54$.

Motic China Group Co., Ltd. (2001-2004). Motic Images Plus version 2.2 Multi langage version. China: Motic China Group.

Moureau, J. (1961). Nouveaux Cordyceps du Congo. Lejeunia, 15, 1-38.

Nikoh, N. y Fukatsu, T. (2000). Interkingdom host jumping underground: phylogenetic analysis of entomoparasitic fungi of the genus Cordyceps. Molecular Biology and Evolution, 17, 629-638.

Nordén, B., Appelqvist, T. y Olausson, B. (2002). Diversity, ecology and conservation of wood-inhabiting ascomicetes in Sweden. Svensk Botanisk Tidskrift, 96, 139-148.

Pérez-Silva, E. (1977). Algunas especies del género Cordyceps (Pyrenomycetes) en México. Boletín de la Sociedad Mexicana de Micología, 11, 145-153.
Pérez-Silva, E. (1978). Nuevos registros de Cordyceps (Pyrenomycetes) en México. Boletín de la Sociedad Mexicana de Micología, 12, $19-25$.

Petch, T. (1933). Notes on entomogenous fungi. Transactions of the British Mycological Society, 18, 48-75.

Quandt, C. A., Kepler, R. M., Gams, W., Araújo, J. P. M., Ban, S., Evans, H. C., et al. (2014). Phylogenetic-based nomenclatural proposals for Ophiocordycipitaceae (Hypocreales) with new combinations in Tolypocladium. IMA Fungus, 5, 121-134.

Rubinoff, D. y Sperling, F. A. H. (2002). Evolution of ecological traits and wing morphology in Hemileuca (Saturniidae) based on a two-gene phylogeny. Molecular Phylogenetics and Evolution, 25, 70-86.

Rubio-Bustos, S., Guzmán-Dávalos, L. y Navarrete-Heredia, J. L. (1999). Especies entomopatógenas de Cordyceps (Fungi Ascomycotina) en México. Boletín del Instituto de Botánica, 7, 135-157.

Sanjuan, T. (2014). Estudio filogenético de la interacción Cordyceps-insectos en el noreste amazónico, un análisis morfológico, molecular y ecológico (Tesis doctoral). Facultad de Ciencias Exactas y Naturales, Universidad de Antioquia: Colombia.

Shimazu, M., Mitsuhashi, W. y Hashimoto, H. (1988). Cordyceps brongniartii sp. nov., the teleomorph of Beauveria brongniartii. Transactions of the Mycological Society of Japan, 29, 323-330.

Shrestha, B., Hyun, M. W., Oh, J., Han, J. G., Lee, T. H., Cho, J. Y., et al. (2014). Molecular evidence of a teleomorph-anamorph connection between Cordyceps scarabaeicola and Beauveria sungii and its implication for the systematics of Cordyceps sensu stricto. Mycoscience, 55, 231-239.

Shrestha, B., Zhang, W., Zhang, Y. y Liu, X. (2012). The medicinal fungus Cordyceps militaris: research and development. Mycological Progress, 11, 599-614.

Sung, J. M. (1996). The insects-born fungus of Korea in color. Seul: Kyohak Publishing Co.

Sung, G. H., Hywel-Jones, N. L., Sung, J. M., Luangsa-Ard, J. J., Shrestha, B. y Spatafora, J. W. (2007). Phylogenetic classification of Cordyceps and the clavicipitaceous fungi. Studies in Mycology, 57, 5-59.

Sung, G. H. y Spatafora, J. W. (2004). Cordyceps cardinalis sp. nov., a new species of Cordyceps with an east Asian-eastern North American distribution. Mycologia, 96, 658-666.

Tuli, H. S., Sandhu, S. S. y Sharma, A. K. (2014). Pharmacological and therapeutic potential of Cordyceps with special reference to Cordycepin. Biotech, $4,1-12$.

Wang, L., Zhang, W. M., Hu, B., Chen, Y. Q. y Qu, L. H. (2008). Genetic variation of Cordyceps militaris and its allies based on phylogenetic analysis of rDNA ITS sequence data. Fungal Diversity, 31, 147-155

Wen, T. C., Li, M. F., Kang, J. C. y He, J. (2012). A molecular genetic study on fruiting-body formation of Cordyceps militaris. African Journal of Microbiology Research, 6, 5215-5221.

Yokoyama, E., Yamagishi, K. y Hara, A. (2005). Heterothallism in Cordyceps takaomontana. FEMS Microbiology Letters, 250, 145-150.

Zang, M. y Kinjo, N. (1998). Notes on the alpine Cordyceps of China and nearby nations. Mycotaxon, 66, 215-230. 